\title{
Medições de uma implementação de MPS.BR nível F
}

\author{
Ítalo B. Borssatto, Adriel R. Moro
}

\author{
Synos Technologies Ltda. \\ Av. Barão Homem de Melo, 4386/14 - 30.450-250 - Belo Horizonte - MG - Brazil \\ \{italo.borssatto,adriel.moro\}@synos.com.br
}

\begin{abstract}
The Synos company, when planning its first software process improvement project, implementing the level F of the MPS.BR, faced the lack of references that could assist it in estimating effort, cost and time. Identifying the chance to supply these references, scarce in the market, the Synos presents its experience, showing the main measurements collected and lessons learned.

Resumo. A empresa Synos, ao planejar seu primeiro projeto de melhoria do processo de desenvolvimento de software, implementando o nível $F$ do MPS.BR, enfrentou a carência de referências que a auxiliassem nas estimativas de esforço, custo e tempo. Identificando a oportunidade de divulgar estas referências, escassas no mercado, a Synos apresenta a sua experiência, revelando as principais medições coletadas e as lições aprendidas.
\end{abstract}

\section{Introdução}

Realizar o primeiro projeto de melhoria do processo de desenvolvimento de software, adequando-o a um nível de maturidade como o nível F do MPS.BR [Weber 2004], é um desafio para qualquer empresa. Uma das principais dificuldades é a falta de relatos de experiência de outras organizações que forneçam insumos para as estimativas durante o planejamento do projeto, pois esse fato prejudica a obtenção do devido apoio da alta direção, uma vez que não são encontradas bases e/ou parâmetros para justificar adequadamente os esforços e investimentos necessários.

A Synos se deparou com essa escassez de referências ao realizar seu primeiro projeto de melhoria. Entretanto, a empresa teve a oportunidade de registrar sua experiência, através de medições e lições aprendidas, e divulgá-la neste artigo. Os esforços e investimentos realizados na implementação do processo de desenvolvimento de software da Synos, o Synos UP, foram registrados utilizando métricas que permitem auxiliar outras empresas que desejem implementar um modelo de maturidade como o MPS.BR nível F.

Para que as medições contidas neste artigo possam ser adequadamente criticadas e analisadas, faz-se necessário demonstrar o contexto em que o projeto de melhoria em questão foi executado. Com este intuito, primeiramente, apresenta-se o resumo de toda a evolução do projeto, com seus marcos e decisões associadas. Em seguida, são reveladas as ferramentas utilizadas no registro e coleta das medições, assim como os valores obtidos e suas análises. E, por fim, as lições aprendidas e as conclusões. 


\section{O projeto de implementação}

A Synos é uma empresa que oferece soluções em tecnologia da informação, cujo corpo técnico é formado por aproximadamente 150 profissionais, dos quais 50 fazem parte da fábrica de software, unidade organizacional avaliada no MPS.BR nível F, em maio de 2007. Seu faturamento, em 2006, foi de aproximadamente 17 milhões de reais, sendo que 1 milhão foi destinado a investimentos internos, como o projeto em questão.

Em março de 2006, a Synos decidiu implementar o nível F do MPS.BR, seguindo o modelo de negócio cooperado definido pela SOFTEX, o MNC-MPS.BR [SOFTEX 2005]. Como instituição implementadora, a Synos contratou a FUMSOFT Sociedade Mineira de Software, utilizando-se de subsídios da SOFTEX.

Antes desta decisão, todos os projetos da empresa seguiam adaptações distintas do RUP [Kruchten 1999], que eram utilizadas de forma deficiente. O Escritório de Gerência de Projetos possuía um processo definido, baseado no PMBOK [Duncan 1996], mas que não estava institucionalizado, e o Escritório de Propostas responsabilizava-se por levantar requisitos, estimar esforços e elaborar propostas técnicas.

A ausência de um processo implantado causava uma série de resultados negativos para a empresa, como a falta de controle dos projetos, atrasos, erros de estimativas de esforço e descontentamento por parte da equipe. Ademais, toda essa estrutura trabalhava fora de sintonia com os esforços de venda da empresa, o que agravava a situação.

O cenário em questão também revelava alguns pontos positivos, mas estava distante dos critérios do MPS.BR nível F. Essa situação foi confirmada no diagnóstico inicial realizado pela Fumsoft e a ASR Consultoria, em maio de 2006, através da verificação de evidências de alguns dos projetos executados pela empresa e entrevistas com a equipe técnica da unidade organizacional. O relatório deste diagnóstico apresentou uma relação detalhada dos pontos fortes e fracos da empresa relativos ao atendimento das áreas de processo do nível 2 do CMMI-SW [Chrissis et al. 2003], equivalente ao nível F do MPS.BR. No resultado, também foi apresentado o interesse da diretoria da empresa na melhoria do processo, o que é essencial para o sucesso de um projeto de melhoria.

Após o diagnóstico inicial, ficou explícita a necessidade da criação de um processo único, aproveitando os já existentes. Desta forma, a Synos estruturou seu projeto de melhoria (vide figura 1) com foco na elaboração de um processo unificado, o Synos UP. 


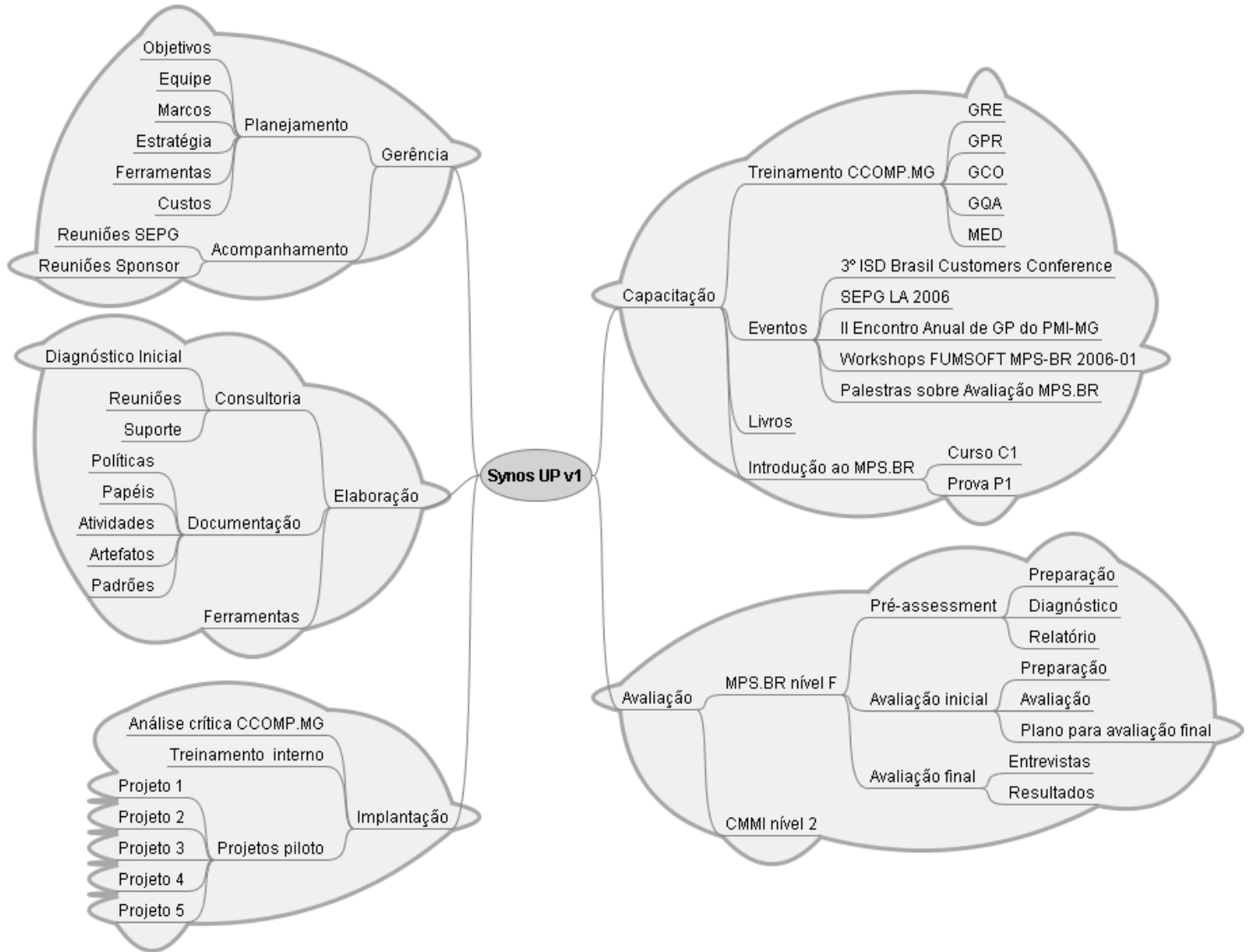

Figura 1. Mapa mental [Buzan 1993] contendo a estrutura do projeto de implementação do processo Synos UP.

A estratégia inicial foi definir cada processo do nível $\mathrm{F}$ utilizando grupos de apoio gerenciados pelo SEPG (Software Engineering Process Group). Estes atuariam paralelamente acelerando a definição do Synos UP e antecipando sua implantação. Dessa forma, a empresa estaria apta a realizar projetos piloto passíveis de eliminação antes da avaliação, trazendo segurança ao projeto de melhoria.

A equipe do projeto, grupos de apoio e $S E P G$, somavam aproximadamente 15 pessoas dedicas parcialmente. Em junho de 2006, uma redução da verba reservada ao projeto inviabilizou esta estratégia, razão pela qual os grupos de apoio foram extintos e o SEPG reduzido a 04 integrantes, sendo 01 dedicado integralmente. A escolha dos membros da equipe foi realizada considerando o conhecimento de cada integrante em relação às áreas de processo do MPS.BR nível F. Neste novo cenário, o tempo para definição do Synos UP prorrogou-se até dezembro de 2006, obrigando a utilização dos projetos piloto na avaliação inicial, algo que a empresa evitava até então.

Em setembro de 2006, a Fumsoft realizou a reunião de Análise Crítica, que objetivava a verificação dos marcos previstos em contrato. Nesta ocasião foram observados resultados aquém do esperado, resultantes da baixa dedicação da equipe, que precisou ter parte de seu esforço alocado em projetos de clientes, seguindo orientação da alta direção. O resultado desta reunião foi um ponto decisivo para o projeto, pois alertou a diretoria em relação à necessidade de maior dedicação da equipe. 
Como conseqüência, a diretoria aumentou o SEPG para 06 integrantes, o que foi mantido até o final do projeto.

Nesse ínterim, na medida em que as definições do processo eram feitas, elas eram imediatamente aplicadas nos projetos em andamento. Concluído o processo, foram realizados 04 projetos piloto, quantidade mínima necessária para uma avaliação de nível F, iniciados entre novembro de 2006 e fevereiro de 2007. As melhorias no processo foram identificadas com mais clareza nesses projetos. Felizmente, as adequações ao modelo MPS.BR puderam ser executadas a tempo, o que foi confirmado pelo diagnóstico de pré-assessment realizado em março de 2007, que seguiu método semelhante ao MA-MPS, mostrando resultado positivo.

A implantação do processo nos projetos piloto foi realizada quando este estava definido, mas não totalmente documentado. Por isso, foi realizado um treinamento antecipado das equipes desses projetos, a fim de viabilizá-la. Cerca de um mês após o treinamento, a versão 1.0 do Synos UP estava disponível no site do processo e, dois meses após o início de sua utilização, foi realizado o treinamento de toda a unidade organizacional, envolvendo cerca de 50 pessoas.

Em 22 e 23 de maio de 2007, a Synos foi submetida à avaliação final do nível F do MPS.BR, concluindo com sucesso o último passo do primeiro projeto de melhoria do processo. Atualmente, a empresa inicia seu segundo ciclo de melhoria, no qual implementará o nível C do MPS.BR.

\section{Medições}

Antes de decidir implementar o MPS.BR nível F, a Synos criou seu SEPG, cuja atividade inicial foi estudar ferramentas que pudessem ser utilizadas no processo de desenvolvimento de software. Além dos registros manuais, as ferramentas que permitiram a coleta das medições apresentadas neste artigo foram: (I) actiTime [actiTime 2007]: ferramenta para apontamento de horas, onde estão registradas as horas trabalhadas por cada membro da equipe; (II) Eclipse Process Framework Composer [EPF 2007]: ferramenta para elaboração de processos; (III) Bugzilla [Bugzilla 2007]: ferramenta de controle de solicitações de mudança e registro de não-conformidades. Outras ferramentas foram utilizadas, porém suas medições não são relevantes para este artigo.

Consultando o apontamento de horas trabalhadas no projeto de melhoria e nos projetos piloto foi possível a geração de gráficos da variação da alocação da equipe e investimento realizados em relação ao tempo (vide figura 2). Relacionando esta distribuição de esforço e custo aos marcos do projeto, descritos no tópico anterior deste documento, podemos analisar os momentos enfrentados:

- Entre a redução da equipe do SEPG e a reunião de análise crítica é possível observar o baixo investimento realizado no projeto, com exceção da participação em um evento e pagamento de uma das parcelas do contrato com a instituição implementadora, o que justifica o resultado negativo observado pela Fumsoft na reunião de análise crítica. 
- Após a reunião de análise crítica, a dedicação ao projeto de melhoria aumentou devido à adição de 02 membros ao SEPG, resultantes da sensibilização da alta direção da empresa frente aos resultados negativos apresentados.

- É nítido o aumento da dedicação ao projeto de melhoria nos últimos meses que antecederam as avaliações, principalmente devido aos treinamentos internos, preparações para avaliações e o esforço realizado nos projetos piloto.

- Os três picos apresentados no gráfico de investimentos estão relacionados às datas de pagamento das parcelas do contrato com a Fumsoft, que inclui a avaliação, consultoria e treinamentos. Este investimento equivale a apenas $25,8 \%$ do investimento total, mostrando que o custo da avaliação é consideravelmente baixo em relação ao investimento total.

- Entre a realização do treinamento interno e o diagnóstico de pré-assessment, a alocação da equipe aumentou. Período em que foi realizado o preenchimento da planilha de avaliação.

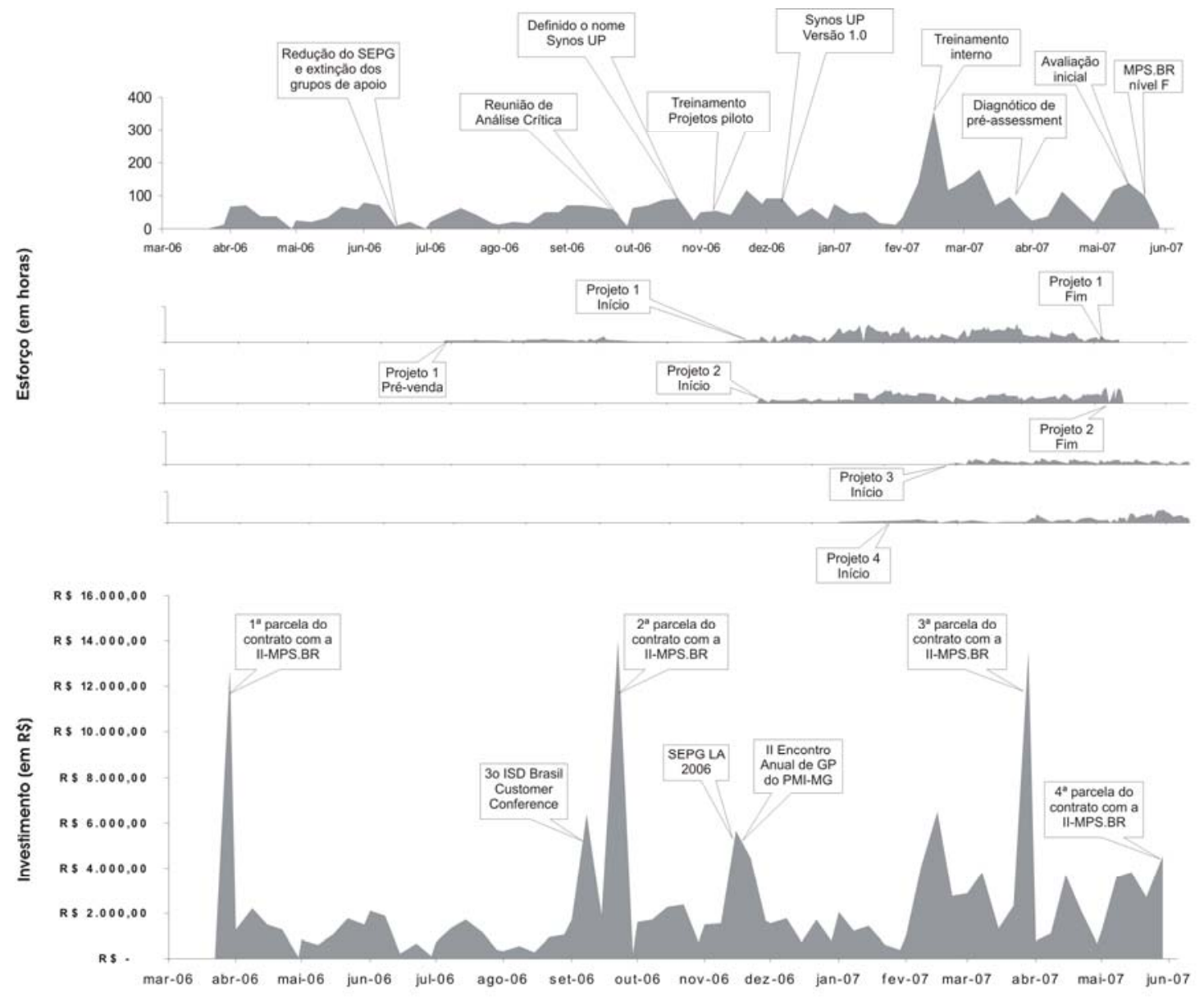

Figura 2. Gráfico da distribuição de esforços e investimentos durante o período do projeto, bem como os respectivos marcos.

O esforço total realizado pela equipe durante o projeto de melhoria foi de 4274,33 horas (veja Tabela 1). Assim, considerando o período de 14,7 meses do projeto, chegamos a um índice de 1,23 pessoas alocadas exclusivamente ao projeto. Estes 
números mostram que a maior parte do investimento realizado neste projeto está relacionada à alocação de recursos humanos.

Tabela 1. Tabela de medições do primeiro projeto de melhoria do processo Synos UP.

\begin{tabular}{|c|c|c|c|c|}
\hline \multicolumn{2}{|l|}{ Período de implementação } & \multicolumn{3}{|l|}{ Investimentos } \\
\hline Data de início & $8 / 3 / 2006$ & Total & $\mathrm{R} \$ 160.787,79$ & $100,0 \%$ \\
\hline Data de término & $23 / 3 / 2007$ & Contrato com Instituição Implementadora & $\mathrm{R} \$ 41.530,00$ & $25,8 \%$ \\
\hline Dias corridos & 380 dias corridos & Documentação do processo (equipe aloc.) & $\mathrm{R} \$ 41.318,36$ & $25,7 \%$ \\
\hline Dias úteis & 273 úteis & Reuniões (equipe alocada) & $\mathrm{R} \$ 27.789,75$ & $17,3 \%$ \\
\hline \multirow[t]{2}{*}{ Meses } & 12,7 meses & Pré-assessment (equipe alocada) & $R \$ 5.566,96$ & $3,5 \%$ \\
\hline & & Diagnóstico inicial (equipe alocada) & $1.165,37$ & $0,7 \%$ \\
\hline \multicolumn{2}{|l|}{ Equipe } & Estudo de ferramentas (equipe alocada) & $6.297,79$ & $3,9 \%$ \\
\hline Quantidade de membros do SEPG & 6 membros & Gerência do projeto (equipe alocada) & $4.207,10$ & $2,6 \%$ \\
\hline Colaboradores na unidade organizacional & 35 colaboradores & Treinamentos (+equipe alocada) & $\mathrm{R} \$ 27.893,81$ & $17,3 \%$ \\
\hline Alocação média de cada membro do SEPG* & $24 \%$ & Avaliação inicial (equipe alocada) & $\mathrm{R} \$ \quad 1.928,88$ & $1,2 \%$ \\
\hline Índice de alocação exclusiva* & 1,42 pessoas & Avaliação final (equipe alocada) & $3.027,27$ & $1,9 \%$ \\
\hline \multicolumn{2}{|l|}{ Esforço } & \multicolumn{3}{|l|}{ Elementos do processo } \\
\hline Esforço total & 4274,33 horas & Atividades & \multicolumn{2}{|c|}{71 elementos } \\
\hline Percentual do esforço da unidade organizacional & $6 \%$ & Artefatos & \multicolumn{2}{|c|}{30 elementos } \\
\hline Esforço do SEPG & 3108,90 horas & Papéis & \multicolumn{2}{|c|}{18 elementos } \\
\hline Por membro do SEPG & 712,39 horas/membro & Checklists & \multicolumn{2}{|c|}{5 elementos } \\
\hline Documentação do processo & 1408,80 horas & Termos técnicos descritos & \multicolumn{2}{|c|}{20 elementos } \\
\hline Treinamento interno & 763,50 horas & Templates & \multicolumn{2}{|c|}{33 elementos } \\
\hline Diagnóstico inicial & 43,5 horas & Guias e tutoriais & \multicolumn{2}{|c|}{23 elementos } \\
\hline Pré-assessment & 109,45 horas & Métricas & \multicolumn{2}{|c|}{46 elementos } \\
\hline Avaliação inicial & 72 horas & & & \\
\hline Avaliação final & 113 horas & \multicolumn{3}{|l|}{ Reuniões } \\
\hline \multicolumn{2}{|l|}{ Percentual do esforço por processo do MPS.BR } & SEPG & \multicolumn{2}{|c|}{46 reuniões } \\
\hline GPR & $29 \%$ & Horas & \multicolumn{2}{|c|}{464,25 horas } \\
\hline GRE & $19 \%$ & Esforço por reunião & \multicolumn{2}{|c|}{10,09 horas } \\
\hline $\mathrm{GCO}$ & $19 \%$ & Sponsor $\star \star \star *$ & \multicolumn{2}{|c|}{15 reuniões } \\
\hline GQA & $12 \%$ & Horas & \multicolumn{2}{|c|}{183,82 horas } \\
\hline MED & $9 \%$ & Esforço por reunião & \multicolumn{2}{|c|}{12,25 horas } \\
\hline Demais processos & $12 \%$ & Consultoria & \multicolumn{2}{|c|}{27 reuniões } \\
\hline \multirow[t]{2}{*}{ Média de horas de treinamento por colaborador } & $21,8 \mathrm{~h} /$ colaborador & Horas & \multirow{2}{*}{\multicolumn{2}{|c|}{389,25 horas }} \\
\hline & & Esforço por reunião & & \\
\hline
\end{tabular}

Vale ressaltar que o pequeno esforço na definição do processo de medição ocorreu em virtude de um dos membros do SEPG ter experiência neste tipo de atividade, o que pode indicar que este desvio pode não ser comum em outras empresas.

É importante observar, também, que os investimentos relacionados diretamente à avaliação estão embutidos no contrato com a instituição implementadora e representa parte pequena de todo o investimento realizado (vide figura 3).

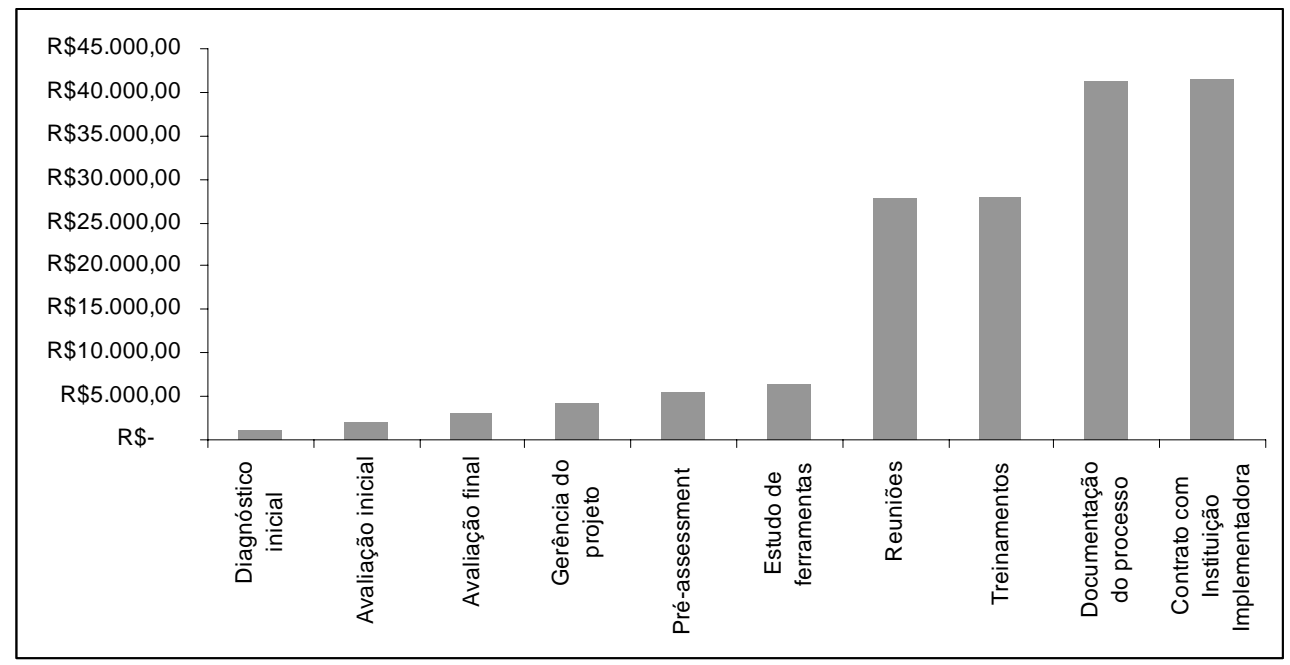

Figura 3. Gráfico da distribuição dos investimentos realizados no projeto de adequação ao nível $\mathrm{F}$ do MPS.BR. 
As medições relacionadas às não-conformidades (NCs) por auditorias de qualidade realizadas nos projetos piloto (veja Tabela 2) ajudam a verificar a eficiência da implantação do processo. No caso da Synos foi obtida uma média de 10,5 NCs por auditoria. A empresa considerou este valor alto, apesar de tê-las corrigido totalmente.

Tabela 2. Tabela de medições relacionadas às auditorias de QA realizadas nos projetos piloto do processo Synos UP.

\begin{tabular}{lc} 
Projetos piloto \\
\hline Total de não-conformidades \\
Não-conformidades por projeto \\
Projeto 1 - BHZ06F014 \\
Auditorias \\
Não-conformidades (total) \\
Não-conformidades por auditoria \\
Projeto 2 - BHZ06F033 \\
Auditorias & $19 \mathrm{NCs}$ \\
Não-conformidades (total) & $6,3 \mathrm{NCs} /$ auditoria \\
Não-conformidades por auditoria & 3 auditorias \\
\end{tabular}

\begin{tabular}{lc}
\hline & \\
& \\
Projeto 3 - BHZ07F004 & \\
Auditorias & 1 auditorias \\
Não-conformidades (total) & $10 \mathrm{NCs}$ \\
Não-conformidades por auditoria & $10,0 \mathrm{NCs} /$ auditoria \\
Projeto 4 - BHZ07F003 & 1 auditorias \\
$\quad$ Auditorias & $16 \mathrm{NCs}$ \\
Não-conformidades (total) & $16,0 \mathrm{NCs} /$ auditoria \\
Não-conformidades por auditoria & $10,5 \mathrm{NCs} /$ auditoria \\
Média de não-conformidades por auditoria &
\end{tabular}

\section{Lições aprendidas}

A realização da implementação do nível F do MPS.BR na Synos foi uma experiência que possibilitou verificar pontos fortes e fracos em sua condução e evolução, quais sejam:

- O prazo de 15 meses, determinado pelo contrato do modelo de negócio cooperado, é desafiador para a implementação do nível F como primeiro projeto de melhoria do processo. $\mathrm{O}$ acréscimo de aproximadamente 3 meses permitiria a aplicação do processo em projetos que não fossem os pilotos.

- A atenção durante a definição dos objetivos do projeto e o foco durante toda a sua execução garantiram o cumprimento dos prazos.

- A realização da reunião de análise crítica apresentou a necessidade de maior apoio da alta direção num dado momento em que a atenção estava sendo deslocada para outros projetos, mostrando a importância do suporte externo oferecido pela consultoria. Sendo certo que, com isso, o apoio da alta direção foi obtido e revelou-se essencial para o bom andamento do projeto, o que foi comprovado pelos valores investidos e a maior dedicação da equipe.

- A alocação de um SEPG relativamente grande, no início do projeto, resultava em reuniões pouco produtivas. Sendo que a redução do grupo, com a dedicação exclusiva de um dos membros do SEPG, manteve o andamento do projeto sem grande impacto.

- A participação de membros do SEPG nos projetos piloto facilitou a implantação do processo.

- A utilização de projetos piloto para a avaliação deve ser evitada, pois apresentam uma quantidade considerável e esperada de não-conformidades.

- A alocação de colaboradores experientes como membros da equipe do SEPG, garantiu a realização do projeto em tempo hábil. Destacando-se o fato de um deles já ter passado por um processo de melhoria similar em outra organização.

- A realização de reuniões quinzenais com a equipe de consultores serviu como um bom ponto de controle para o andamento do projeto, assim como a 
constância com que o grupo se reuniu para discutir o processo contribuiu para sua rápida definição.

- A participação em eventos ( $3^{\circ}$ ISD Brasil e SEPG-LA), aquisição de livros e realização de treinamentos colaborou fortemente na implantação do processo;

- A realização de um treinamento interno envolvendo todos os colaboradores, inclusive os que não participariam do processo, acelerou a sua institucionalização.

- Os processos de qualidade e medição são extremamente importantes no auxílio à implantação do processo e devem ser atualizados à medida que novas práticas são adicionadas ao processo. A pouca dedicação a estes dois processos no início do projeto prejudicaram sua implantação.

\section{Conclusão}

A implementação de um modelo de maturidade como o MPS.BR nível $\mathrm{F}$ exige um investimento em alocação de recursos humanos que supera, consideravelmente, os custos da avaliação MA-MPS. Portanto, a conscientização da alta direção em relação a esta característica do projeto é necessária para que o devido apoio seja oferecido, dando segurança à execução do projeto, adiantando sua implantação e permitindo que outros projetos de desenvolvimento de software, além dos pilotos, sejam executados antes da avaliação final.

\section{Referências}

Weber, K., Rocha, A. R. et al. (2004) "Modelo de Referência para Melhoria de Processo de Software: uma abordagem brasileira”, In: Anais da XXX Conferência Latinoamericana de Informática (CLEI2004), Arequipa, Peru.

SOFTEX (2005), "Modelo de Negócio para Melhoria de Processo de Software (MNMPS.BR)”, v100605. Disponível em http://www.softex.br/mpsbr/, em 15/03/2007.

Kruchten P. (1999). The Rational Unified Process: An Introduction. Addison Wesley

Chrissis, M. B., Konrad, M, Shrum, S. (2003) “CMMI: Guidelines for Process Integration and Product Improvement”, Addison-Wesley.

Buzan, T. (1993) The Mind Map Book. London: BBC Books

Freire, Herval. (2003) “Calculando Estimativas: o Método de Pontos de Casos de Uso”. Disponível em http://www.cnnt.com.br/files/usecasepoints.pdf, em 15/03/2007.

Duncan, W. R. (1996), A Guide to the Project Management Body of Knowledge, PMI Standards Committee, Program Management Institute, 1996.

actiTime (2007), Free Web Based Time Tracking Software. Disponível em http://www.actitime.com/, em 15/03/2007.

Bugzilla (2007), disponível em http://www.bugzilla.org/, acessado em 15/03/2007.

EPF (2007), Eclipse Process Framework Composer. Disponível em http://www.eclipse.org/epf/, em 15/03/2007. 